\title{
Diagnosis of Babesiosis by Conventional Microscopy and PCR
}

\author{
B. Sai Sindhu ${ }^{1 *}$, B. Shobhamani ${ }^{1}$, K. Suresh ${ }^{2}$ and V. Chengalva rayulu ${ }^{3}$ \\ ${ }^{1}$ Department of Veterinary Medicine, ${ }^{3}$ Department of Veterinary Parasitology, \\ College of Veterinary Science, Tirupati, India \\ ${ }^{2}$ Department of Veterinary Clinical Complex, N.T.R College of Veterinary Science, \\ Gannavaram, India \\ *Corresponding author
}

\section{A B S T R A C T}

\section{Ke y w o r d s \\ Babesia gibsoni, Babesia canis, Diagnosis, Microscopy, Polymerase chain reaction \\ Article Info \\ Accepted: 04 August 2020 Available Online: 10 September 2020}

The present study was conducted in Veterinary Clinical Complex, College of Veterinary Science, Tirupati over a period of nine months to diagnose the Babesia infection. 235 dogs suspected for babesiosis were collected and subjected for diagnosis using conventional and molecular techniques. Out of 235 samples examined by conventional microscopy of Giemsa stained blood smears revealed eleven positive samples which includes Babesia gibsoni (10) and Babesia canis (1) whereas with polymerase chain reaction 20 samples showed amplicons of $671 \mathrm{bp}$ and 3 samples showed amplicons of 450 bp indicative of Babesia gibsoni and Babesia canis respectively.

\section{Introduction}

Babesiosis is one of the important tick-borne haemoprotozoan disease caused by the species of genus Babesia. Babesia species belong to the phylum Apicomplexa, class Piroplasmea, order Piroplasmida and family Babesiidae. Canine babesiosis is a clinically significant and geographically wide spread disease of domesticated dogs and wild canids (Irwin 2009). Multiple species of Babesia have been documented to infect dogs with babesiosis. However, Babesia canis and
Babesia gibsoni are the only species found to be present in Asia. Babesia canis is a large form of piriform (tear drop shaped) organism with 2.5-5.0 $\mu \mathrm{m}$ in size, whereas small pleomorphic organisms of size 1.0-2.5 $\mu \mathrm{m}$, which appears as oval or signet ring form are termed as Babesia gibsoni (Conrad et al., 1991 and Solano-Gallego et al., 2011). Babesia spp. are transmitted by variety of ticks including brown dog tick (Rhipicephalus sanguineus), and different members of Dermacenter genus. Transmission can also occur from dog to dog through bite (Jefferies 
et al., 2007) and transplacental transmission (Fukumoto et al., 2005). Clinically canine babesiosis has been found to result in a wide range of presentations from sub clinical to serious illness characterised by fever, depression, pallor, jaundice, lymphadenopathy, spleenomegaly, weakness and collapse associated with intravascular and extravascular haemolysis, hypoxic injury, systemic inflammation, thrombocytopenia. After initial acute infection, the animal may become a chronic carrier (Irwin, 2009). Therefore, the diagnosis of this disease and the detection of dogs that are carriers or that have a chronic form of this disease are very important.

Currently definitive means of diagnosis of canine babesiosis is based on demonstration of the parasite in Giemsa stained thin-film blood smears examined by microscopy. However, the detection of Babesia parasites is difficult in dogs with unapparent or chronic infections since the parasitemia is very low. Therefore the development of highly specific and sensitive methods is required for diagnosis of infection. It has become possible to detect infection with molecular techniques like PCR and supporting haematological and biochemical findings which can correlate the pathological process of the disease.

Along with these tests various serological methods such as complement fixation test (CFT), immunofluorescent antibody test and enzyme linked immunosorbent assay (ELISA) were used for diagnosis. polymerase chain reaction (PCR) assay present a higher sensitivity and specificity than the blood smear evaluation and may differentiate species that may not be morphologically distinguished by smear method (Boozer and Macintire, 2003). In the present study the diagnosis of babesiosis in dogs was done by examination of giemsa stained blood smear and PCR methods.

\section{Materials and Methods}

\section{Blood samples}

During the period under study (December 2018 to August 2019), a total number of 235 dogs exhibited clinical signs suggestive of haemoprotozoan infection such as pyrexia, pale mucous membranes, anorexia, lymphnode enlargement, weakness and lethargy were selected and screened for babesiosis. The dogs were subjected for routine clinical laboratory evaluation including peripheral blood smear examination and PCR. A thin blood smears from the suspected dogs were prepared and then fixed in methanol for 1 minute and stained with Giemsa (1:6) solution for 30 minutes as described by Fukumoto et al., (2001). Then the slide was examined for Babesia organisms with light microscope under 100X magnification. Blood samples were taken into EDTA tubes and kept at $-20^{\circ} \mathrm{C}$ for upto further confirmation by PCR assay.

\section{DNA extraction and PCR assay}

DNA was isolated from $200 \mu \mathrm{l}$ amounts of EDTA blood from each dog using QIAamp DNA blood mini kit (QIAGEN, Hidden, Germany) according to the manufacturer's instructions. The primer sequence used for Babesia canis were forward primer PIROA (5'AGGGAGCCTGAGAGACGGCTACC

$\left.3^{\prime}\right)$ and reverse primer PIRO-B (5' TTAAATACGAATGCCCCCAAC 3') were used to amplify an approximately at $450 \mathrm{bp}$ region of the $18 \mathrm{~S}$ rRNA gene (Foldavari et al., 2005) and for Babesia gibsoni the primer sequence was forward primer Gibb 599 (5', CTCGGCTACTTGCCTTGTC 3') and reverse primer (5, GCCGAAACTGA AATAACGGC 3') were used to amplify an approximately at $671 \mathrm{bp}$ region of $18 \mathrm{~s}$ rRNA gene (Inokuma et al., 2004). The reaction mixture components i.e., nuclease free water, 
DNA template, forward and reverse primers (10pM), and master mix and their quantities were standardized for 18S rRNA gene of Babesia canis and Babesia gibsoni. The amplification of DNA was carried out in thermal cycler (Proflex PCR, applied biosystems by life technologies, Singapore). For species specific DNA of Babesia canis the thermocycle profile consisted of initial denaturation at $94^{\circ} \mathrm{C}$ for ten minutes, followed by 40 cycles of denaturation at $94^{\circ} \mathrm{C}$ for thirty seconds, annealing at $60^{\circ} \mathrm{C}$ for thirty seconds, extension at $72^{\circ} \mathrm{C}$ for thirty seconds and this was followed by a final extension at $72^{\circ} \mathrm{C}$ for five minutes.

For species specific DNA of Babesia gibsoni the thermocycle profile consisted of initial denaturation at $95^{\circ} \mathrm{C}$ for five minutes, followed by 35 cycles of denaturation at $95^{\circ} \mathrm{C}$ for thirty seconds, annealing at $55^{\circ} \mathrm{C}$ for thirty seconds, extension at $72^{\circ} \mathrm{C}$ for ninety seconds and this was followed by a final extension at $72^{\circ} \mathrm{C}$ for five minutes. Amplified DNA was subjected to electrophoresis in a $1.5 \%$ agarose gel prestained with ethidium bromide and viewed under Gel doc (BioRad, Dell, USA). The size and specificity of PCR products were confirmed by comparing with the $100 \mathrm{bp}$ DNA ladder. Negative control with distilled water was maintained in each PCR.

\section{Results and Discussion}

Out of 235 samples screened by peripheral blood smear examination of smears by Giemsa staining, 11 samples were found positive for Babesia spp. among these positive samples ten were found positive for Babesia gibsoni and one sample was found positive for Babesia canis. The identification was done based on the characteristic morphology of the organisms. Babesia gibsoni appeared as pleomorphic oval or signet ring (Fig. 1) while Babesia canis appeared as piriform shaped (tear drop) either singlet or paired form (Fig.2). PCR showed greater sensitivity in diagnosing infection than microscopy 23 samples were found positive which includes eleven microscopically positive samples Out of these 23 positive samples, 20 samples showed amplicons of $671 \mathrm{bp}$ and 3 showed amplicons of $450 \mathrm{bp}$ which are suggestive of Babesia gibsoni and Babesia canis, respectively (Fig.3,4).

Giemsa stained blood smear examination findings in the present study corroborates with the results of Praveen et al., (2015), Vipan et al., (2015) and Patra et al., (2018). In the present investigation Babesia gibsoni was the predominant species in this region which was in agreement with the earlier observations of Das et al., (2015) and Kumar et al., (2009) who reported higher prevalence of Babesia gibsoni in their study.

Microscopic examination remains the simplest, commonly used economically feasible, most accessible rapid confirmatory diagnostic method for identification of Babesia spp. which is frequently used under field conditions as it is conclusive. Examination of stained peripheral blood smear was considered as excellent diagnostic tool for acute infections but not suitable to diagnose asymptomatic carriers due to low level of parasitemia (Ayoob et al., 2010 and Bano and Lodh, 2018).

In the present study PCR detected more number of samples as compared to peripheral blood smear examination indicating higher sensitivity and specificity of PCR which corroborates with the findings of Mahalingaiah et al., (2017) and Jain et al., (2017) and they opined that subclinical / chronic status of the infection can be detected by PCR. 
Fig.1 Intraerythrocytic pirolasms of Babesia gibsoni
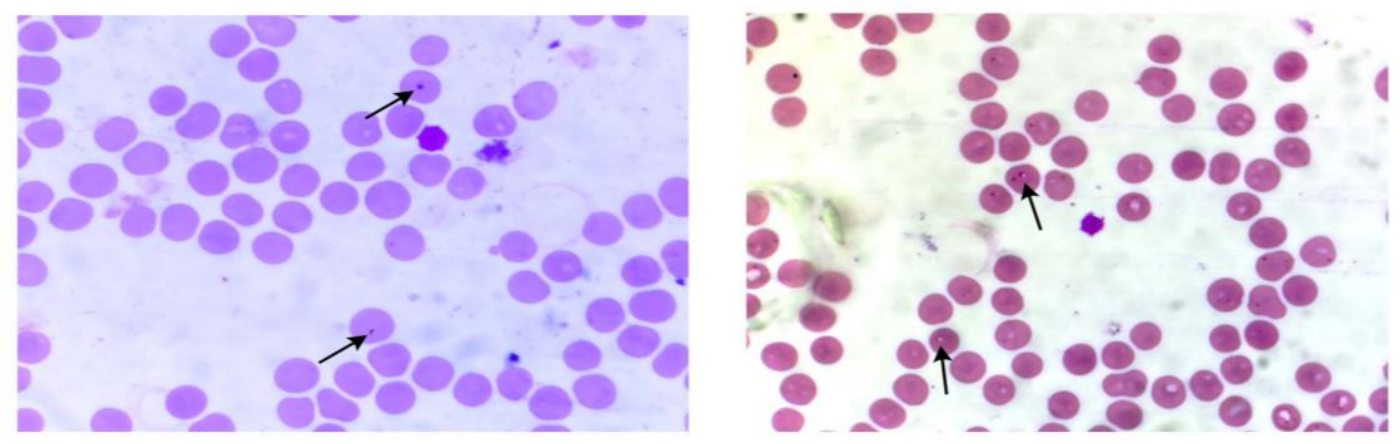

Fig.2 Intraerythrocytic pirolasms of Babesia canis
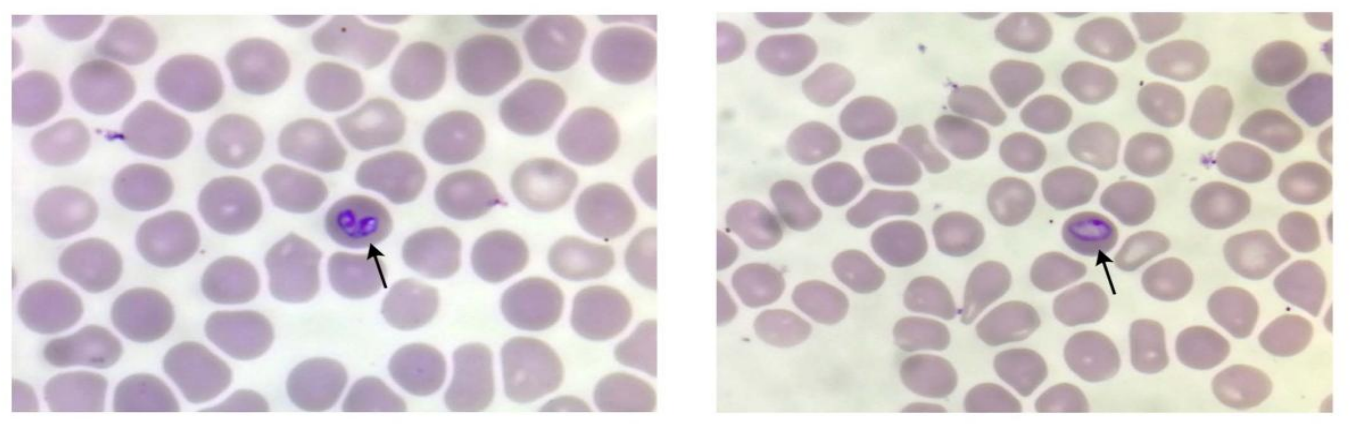

Fig.3 Babesia gibsoni positive samples by PCR

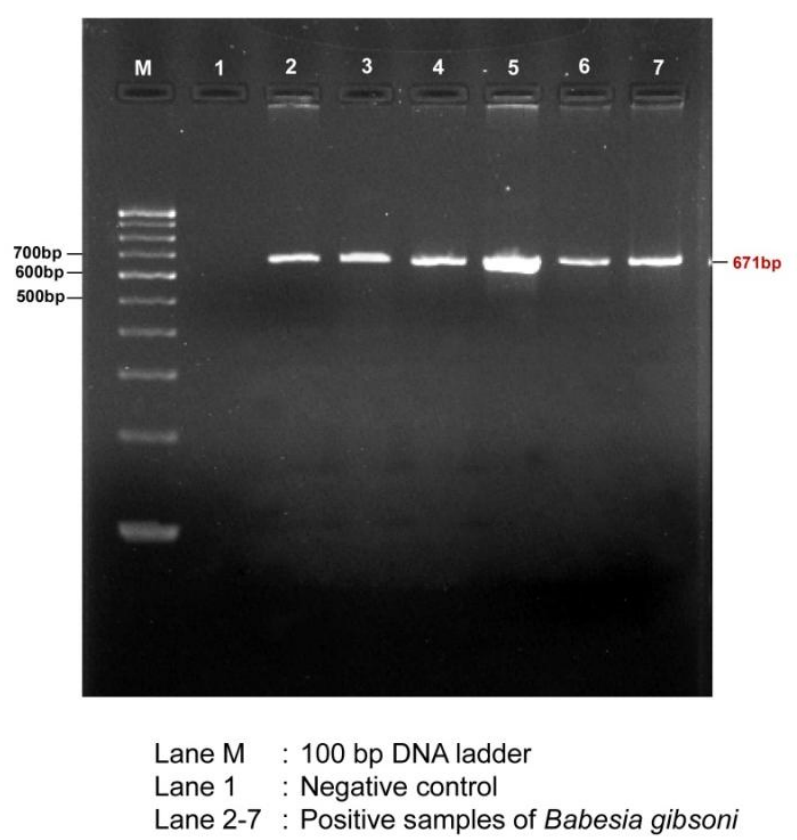


Fig.4 Babesia canis positive samples by PCR

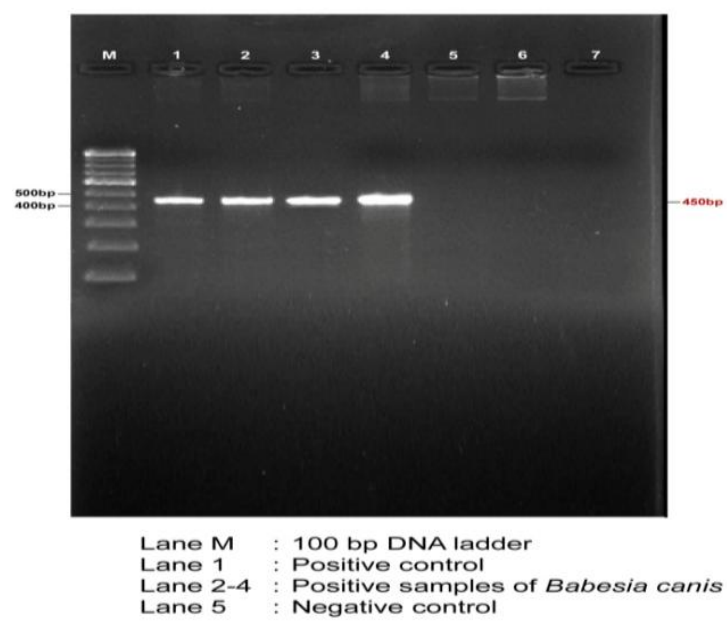

PCR is the most sensitive and specific test available for diagnosis of Babesia infection. This methodology targets parasitic DNA rather than anti-babesial antibodies, and is therefore a reliable diagnostic tool in per acute, acute, and chronic infections. It is capable of diagnosing babesial infections with small volume of blood sample having extremely low parasitemia. PCR is commonly used in clinical diagnosis, identification of new strains, and to differentiate alike and genetically distinct Babesia spp. in epidemiologic studies as endorsed by Ayoob et al., (2010) and Inokuma et al., (2004).

Other diagnostic methods available for detection of Babesia spp. were flow cytometry, indirect fluorescent antibody test (IFAT), enzyme linked immuno sorbent assay (ELISA) etc., although these are effective for detection of antibodies to Babesia spp., crossreactivity occurs between Babesia spp., Toxoplasma spp., and Neospora spp. infections and they fail to differentiate current and past infections (Ayoob et al., 2010 and Dantas-Torres and Figueredo, 2006).

In conclusion, hence proper control measures for ectoparasitic should be followed in dogs. Utility of multiple diagnostic tests is suggested for confirmatory detection and epidemiological disease investigations of canine babesiosis in dogs. Molecular techniques like PCR were highly sensitive method for diagnosis of babesiosis infection in dogs as it detects low infection or chronic stage of infection when compared to conventional microscopy.

\section{Acknowledgements}

The authors are thankful to college of veterinary science, Tirupati, department of veterinary medicine for providing financial assistance and facilities provided to carry out the research work.

\section{References}

Ayoob, A L., Hackner, S.G., and Prittie, J. (2010). Clinical management of canine babesiosis. Journal of Veterinary Emergency and Critical Care. 20 (1): 77-89.

Bano, S. and Lodh, C., (2018). Diagnosis and management of Canine Babesiosis. Intas Polivet. 19 (II): 245-248.

Boozer, A.L., and Macintire, D. K., (2003). Canine babesiosis. Veterinary Clinics of North America: Small Animal Practice. 33: 885-904.

Conrad, P., Thomford, J., Yamane, I., Whiting, J., Bosma, L., Uno, T., Holshuh, H.J. and Shelly, 
S. (1991). Hemolytic anemia caused by Babesia gibsoni infection in dogs. Journal of American Veterinary Medicine Association. 199 (5): 601-605.

Dantas-Torres, F. and Figueredo, L.A. (2006). Canine babesiosis: A Brazilian perspective. Veterinary Parasitology. 141: 197-203.

Das, M.K., Baidya, S., Mahato, A., Pandit, S., Ghosh, J.D., Chaudhuri, S. and Das, M. (2015). Incidence of canine babesiosis in and around Kolkata, West Bengal, India. Exploratory Animal and Medical Research. 5 (1): 102-107.

Fukumoto, S., Suzuki, H., Igarashi, I. and Xuan, X. (2005). Fatal experimental transplacental Babesia gibsoni infections in dogs. International Journal of Veterinary Parasitology. 35: 1031-1035.

Fukumoto, S., Suzuki, H., Igarashi, I. and Xuan, X. (2005). Fatal experimental transplacental Babesia gibsoni infections in dogs. International Journal of Veterinary Parasitology. 35: 1031-1035.

Fukumoto, S., Xuan, X., Shigeno, S., Kimbita, E., Igarashi, I., Nagasawa, H., Fujisaki, K., and Mikami, T., (2001). Development of a polymerase chain reaction method for diagnosing Babesia gibsoni infection in dogs. Journal of Veterinary Medical Science. 63: 977-981.

Inokuma, H., Yoshizaki, Y., Matsumoto, K., Okudaa, M., Onishi, T., Nakagome, K., Kosugi, R. and Hirakawa, M. (2004). Molecular survey of Babesia infection in dogs in Okinawa, Japan. Veterinary Parasitology. 121: 341-346.

Irwin, P.J., Parasitology. Vector, (2009). 2: 4

Jain, K.J., Lakshmanan, B., Syamala, K., Praveena, J.E. and Aravindakshan, T. (2017). High prevalence of small Babesia species in canines of Kerala, South India. Veterinary
World. 10 (11): 1319-1323.

Jefferies, R., Ryan, UM. and Jardine, J. (2007). Blood, bull terriers and babesiosis: further evidence for direct transmission of Babesia gibsoni in dogs. Aust Vet J; 85(11):459-463.

Kumar, K.S., Vairamuthu, S. and Kathiresan, D. (2009). Prevalence of haemoprotozoans in canines in Chennai city. Tamilnadu Journal of Veterinary \& Animal Sciences. 5 (3): 104-108.

Mahalingaiah, M.K.C., Asoor, M., Thimmaiah, R.P., Narayanaswamy, H.D., Mukartal, S.Y., Elattuvalappil, A.M., Chikkahonnaiah, N., Gupta, S. and Singh, S. (2017). Prevalence of Canine Babesiosis in Different Breeds of Dogs in and Around Bengaluru. Advances in Animal and Veterinary Sciences. 5 (3): 140 144.

Patra, G., Sahara, A., Ghosh, S., Behera, P., Borthakur, S.K., Biswas, P., Debbarma, A. and Alam. S.S. (2018). Prevalence of tickborne pathogens in domestic dogs in NorthEastern region of India. Biological Rhythm Research. DOI: 10.1080/09291016.2018.1526495.

Praveen, K., Kumar, V., Gopal, H., Sharma, H. and Wadhawan, V.M. (2015). Prevalence of canine babesiosis in and around Jalandhar (Punjab), India. Veterinary Clinical Science. 3 (1): 1-3.

Solano-Gallego, L. and Baneth, G. (2011). Babesiosis in dogs and cats-Expanding parasitological and clinical spectra. Journal of Veterinary Parasitology. 181: 48-60.

Vipan, K., Parvinder, K., Charanjeet, S., Heigo, P., Gagandeep, B., Hanish, S. and Wadhawan, V.M. (2015). Prevalence of Canine Babesiosis in Jalandhar District, Punjab, India. Research Journal of Animal, Veterinary and Fishery Sciences. 3 (4): 6-8.

\section{How to cite this article:}

Sai Sindhu, B., B. Shobhamani, K. Suresh and Chengalva Rayulu, V. 2020. Diagnosis of Babesiosis by Conventional Microscopy and PCR. Int.J.Curr.Microbiol.App.Sci. 9(09): 345350. doi: https://doi.org/10.20546/ijcmas.2020.909.044 